\title{
Improved Image Processing Techniques for User Immersion Problem Alleviation in Virtual Reality Environments
}

\author{
Dr. R. Dhaya \\ Professor, \\ Department of Computer science and Engineering \\ King Khalid University \\ Kingdom of Saudi Arabia \\ dhayavel2005@gmail.com
}

\begin{abstract}
The latest advertisements on the advancements of the virtual reality has paved way for diverse studies, in manifold fields that can benefit by utilizing the technologies of the virtual reality, not excluding the design, gaming and the simulated understanding. Yet whenever a virtual reality device conveys information in form of images with the assistance of the display that is positioned closer to the user's eyes it faces problems like minimizing the speed of the process and degradation in the quality of images ending up in huge variations across the virtual realism and the realism causing user immersion problems. So to mitigate the immersion problems of the user because of the low quality of image and the minimization of processing speed in the virtual reality environments the paper puts forth an improved image processing technique to improvise the sharpness of the images in order to enhance quality of the images and heighten the processing speed.
\end{abstract}

Keywords: Image Processing Technique, Virtual Reality, Reality, Sharpness, User Immersion Problem.

\section{Introduction}

The latest advertisements on the advancements of the virtual reality has paved way for diverse studies, in manifold fields that can benefit by utilizing the technologies of the virtual reality, not excluding the design, gaming and the simulated understanding. The technologies of virtual reality applied over diverse field cause innovative and novel supplementary values to our regular way of living. Yet while conveying the information in the form of images using the closely positioned displays the virtual reality experiences major problems such as speed minimization of the process and the degradation in the quality of the images causing user immersion difficulties as huge difference prevails across the real and the virtually real images.

So to mitigate the difficulties' in the user immersion the paper puts forth the enhancement algorithm to improve the processing of images ending in images with better sharpness and higher processing speed. The 
Journal of Innovative Image Processing (JIIP) (2020)

Vol.02/ No. 02

Pages: $77-84$

https://www.irojournals.com/iroiip/

DOI:_https://doi.org/10.36548/jiip.2020.2.002

main scope of the proposed work is to enhance the quality of the images transmitted from the virtually real environments and to minimize as well as resolve the immersion difficulties by minimizing the gap across the actual and the virtually real images.

The proposed method utilizes the "Retinex algorithm to enhance the quality of the images and with the assistance of the nearby functions and the weighted average of the neighboring functions and the Otsu's algorithm to maximize the processing speed. The proposed method with the image quality enhancement and the user immersion difficulties alleviation is arranged with the section 2 presenting the previous works, and the related works, the section 3 with the proposed algorithm on image quality enhancement, section 4 on performance evaluation section 5 presenting the summary of the work done.

\section{Related Works}

Robertson, et al [1] conducted works incorporating the virtual reality in the desktop and the step in alleviating the immersion problems. Bowman et al [2] elaborated methodologies to understand the level of immersion required for the virtual reality. Weiss et al [3] applied the virtual reality in works like VRrepresentation, visualization education, distance communication, training, orientation and navigation. Carrozzino, et al [4] devised the mechanism to segregate the virtually real configurations, that are especially linked with the cultural heritage applications.

Akizuki et al [5] elaborated the "Effects of immersion in virtual reality on postural control." Burdea, et al [6] this book discusses the importance of the virtual reality technology in diverse fields. Fehn, et al [7] proposed techniques to perform "Depth-image-based rendering (DIBR), compression, and transmission for a new approach on 3D-TV." Urbancsek, et al [8] performed the "Sensing and Monitoring Methods in Telerobotical Applications Fusing Real-time Video Transmission and Calibrated Virtual Reality."Bindhu, V. et al [9] conducted the Biomedical Image Analysis Using Semantic Segmentation."

Lee, et al [10] devised the algorithm incorporating the ostu's segmentation to improve the processing speed.Song et al [11] conducted the "Regulatory reform solution of VR contents industry based on simulator." The "comprehensive review, enhancements and research directions in the Image and audio fractal compression" was put forth by Ali, et al [12]

Shakya, Subarna et al [13] proposed the "Virtual Restoration of Damaged Archeological Artifacts Obtained from Expeditions Using 3D Visualization."Wen, et al [14] put for the multi scale Retinex based image enhancement for the Autonomous robot navigation in the environments with low light. Zhang et al [15] 
Journal of Innovative Image Processing (JIIP) (2020)

Vol.02/ No. 02

Pages: $77-84$

https://www.irojournals.com/iroiip/

DOI:_https://doi.org/10.36548/jiip.2020.2.002

utilized Retinex to identify the plants in the underwater Manoharan, S et al [16] devised "A Smart Image Processing Algorithm for Text Recognition, Information Extraction and Vocalization for the Visually Challenged."

\section{Image Quality and Processing Speed Enhancements in Virtually Real Environment}

The improved image processing algorithm proposed in the paper scopes in improving the image quality and heightening the processing speed. For this purpose the proposed algorithm utilizes the multi scale retinex algorithm [14] and the approximate threshold estimation in Ostu's [10]. Highly compatible speed for computing the images for the virtually real applications is sixteen milliseconds. The decrease in the computing speed would increase the gap across the real and the virtually real images. The quality degradation in the images is a result of reduced sharpness in the images convey form the virtual reality. So the laid out protocol eliminates the unnecessary information hiding in the images to improve the sharpness of the images. The input image is compressed and subjected to the multi scale Retinex (MSR), to improve the quality of the image. The MSR extricates the original image eluding the unwanted components employing the Gaussian Filter. The equation 1 is framed in this regard.

$$
\iint G(u, v) d u d v=I_{I}
$$

Where $I_{I}$ is the input image and the $G$ is the Gaussian filter. The outcomes observed as result of applying the equation is normalized to value ranging from 0 to 255, the neighboring functions (Gaussian Filter) and the weighted average of the neighboring function to improve the quality of the images. The equation 2 is framed in this regard.

$$
\operatorname{MSR}(u, v)=\sum_{n=1}^{N} w_{n} \log I_{I}(\mathrm{u}, \mathrm{v})-\log \left[G_{n}(u, v) * I_{n}(\mathrm{u}, \mathrm{v})\right]
$$

Using this above method the data that are light source are extricated by acquiring a three dimensional image. Alterations in the data is done using the weights functions that improves the sharpness by applying the weights. This balances the luminous in the darkness as well as the bright areas in the image. The weights also eludes the "halo effect". This estimates the boundaries of every images and determines the segmented boundaries of the images, the actual weight estimated is applied to every border. The weight are estimated using the Ostu's segmentation.

The speed of the processing images is estimated by the elements of the images observed in the output in particular duration. To enhance the processing speed the unwanted image components are removed. 
Journal of Innovative Image Processing (JIIP) (2020)

Vol.02/ No. 02

Pages: $77-84$

https://www.irojournals.com/iroiip/

DOI:_https://doi.org/10.36548/jiip.2020.2.002

Though more promising techniques such as the binarization etc. can be used. The proposed method uses the Ostu protocol as it affords in providing a high processing speed in computing the images. The following equations 3 and 4 shows the process of the Ostu in determining the weights.

$$
\operatorname{class}^{\wedge} 2_{\text {weight }}(t)=\text { weight } 1(2) \operatorname{Class}^{\wedge} 2_{1}(t)+\text { weight } 2(t) \operatorname{Class}^{\wedge} 2_{2}(t)
$$

Weight $1(t)$. weight $2(t)[\mu 1(t)-\mu 2(t)]^{\wedge} 2$

From the above equations the weight 1 and weight 2 are the probability across the two segregated classes class 1 and class 2 . The "inter- class variance maximization is equivalent to its minimization. The image data size and processing speed could be enhanced by the eliminating the extricated image elements. The figure. 1 below shows the stages of the proposed image computing technique".

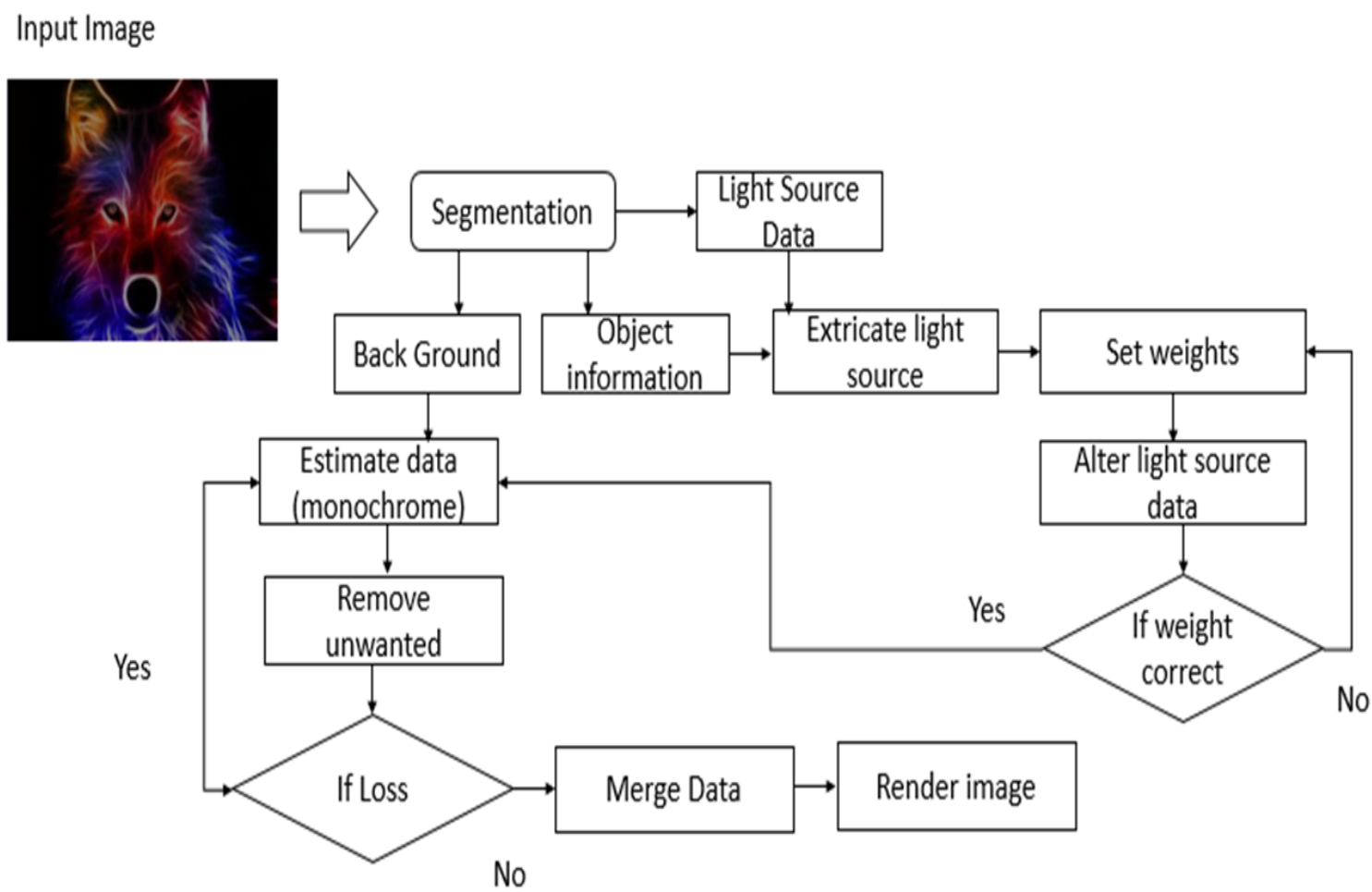

Figure.1 Proposed Image processing Technique. 
Journal of Innovative Image Processing (JIIP) (2020)

Vol.02/ No. 02

Pages: $77-84$

https://www.irojournals.com/iroiip/

DOI:_https://doi.org/10.36548/jiip.2020.2.002

\section{Performance Evaluation}

The proposed algorithm is evaluated with the set of images in the MATLAB to estimate the sharpness and the processing speed of the images. The virtually real images are comprised with the huge set of data. The algorithm operation time and the allotted CPU part increases when the traditional enhancement methods and for enhancing the quality and computing the images are applied. This was observed as the result of evaluation. The sample image used in testing the proficiency of the algorithm proposed is depicted below in the figure. 2
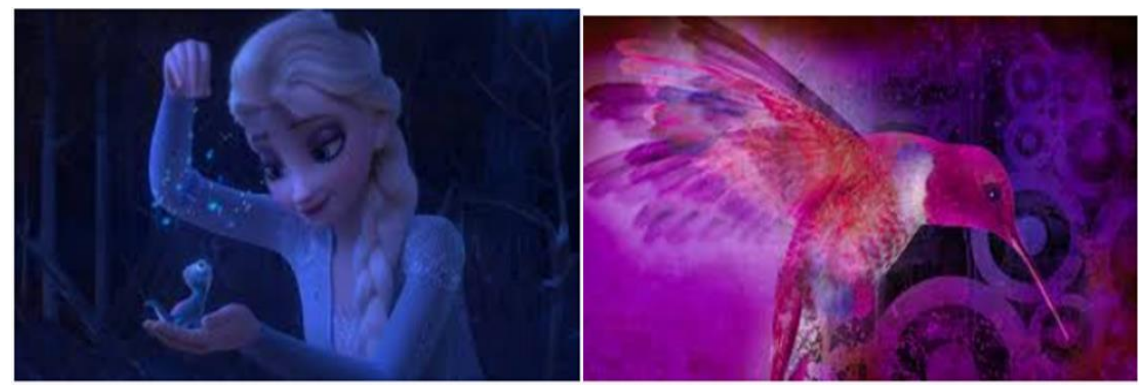

Real images
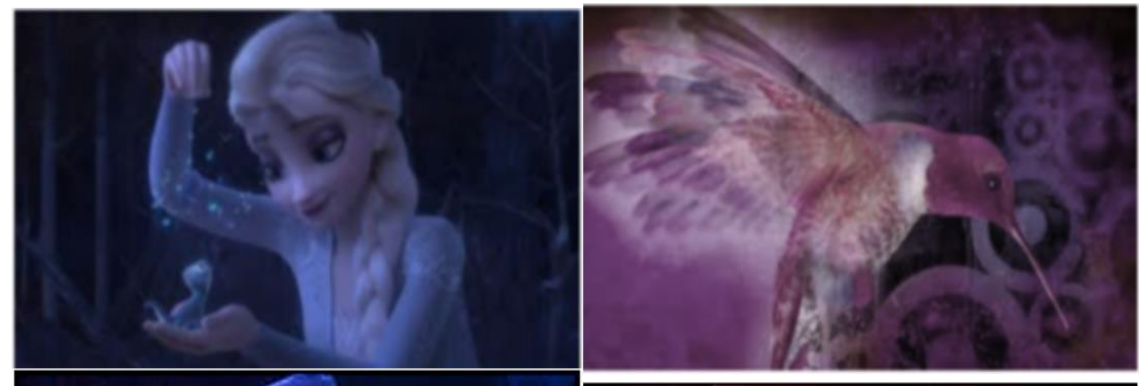

Out comes of conventional algorithm
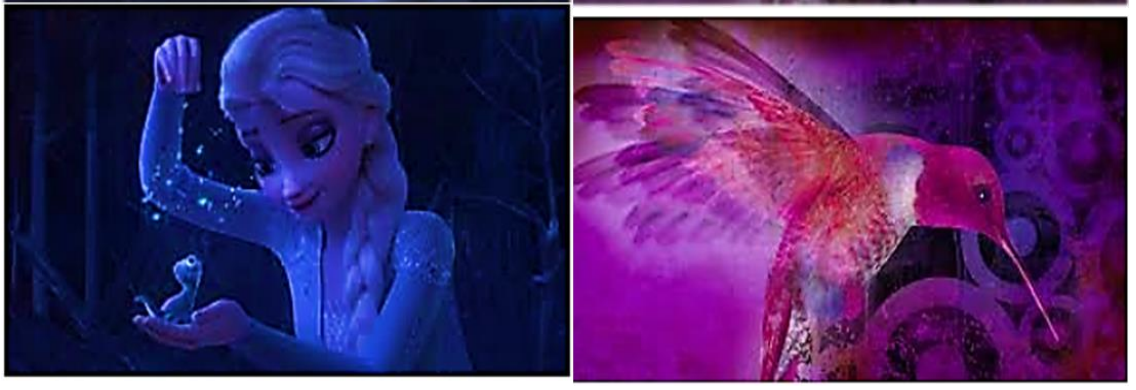

Out comes of Proposed

Figure.2 Sample Image and Outcomes

The laid out protocol accepts the three dimensional images and eludes the necessary data by identifying and correlating the grey scale images. The grey scale data ranges from five to ten frames per second, so the losses remains hidden while eliminating the gray scale information's. The identification of the grey scale

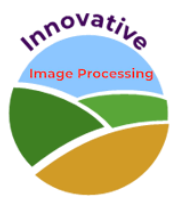


Journal of Innovative Image Processing (JIIP) (2020)

Vol.02/ No. 02

Pages: $77-84$

https://www.irojournals.com/iroiip/

DOI:_https://doi.org/10.36548/jiip.2020.2.002

does not have any impact over the computing speed and the quality of the images. The concurrent enhancement is shown in the computing speed and the image quality by applying the proposed protocol. Finally the images are solidified and delivered at the output. The table. 1 below shows the $\%$ of sharpness and the \% image quality observed on the application of the proposed and the conventional algorithms. The gap across the real and the virtually real images are also listed in the table. For different number of images used in evaluating the proposed method.

\begin{tabular}{cccccccc}
\hline \multicolumn{1}{c}{ Images } & \multicolumn{3}{c}{ Conventional } & \multicolumn{3}{c}{ Proposed } \\
\cline { 2 - 8 } & Sharpness \% & $\begin{array}{c}\text { Image } \\
\text { quality \% }\end{array}$ & $\begin{array}{c}\text { Gap across } \\
\text { Real and } \\
\text { virtually real }\end{array}$ & Sharpness \% & $\begin{array}{c}\text { Image } \\
\text { quality \% }\end{array}$ & $\begin{array}{c}\text { Gap across } \\
\text { Real and } \\
\text { virtually real }\end{array}$ \\
\hline Image set 1 & -55 & 75 & .65 & 50 & 85 & .0345 \\
Image set 2 & -43 & 67 & .55 & 40 & 88 & .0245 \\
Image set 3 & -23 & 65 & .78 & 45 & 89 & .0189 \\
Image set 4 & -49 & 78 & .79 & 49 & 90 & .0112 \\
Image set 5 & -45 & 69 & .58 & 55 & 92 & .0014 \\
\hline
\end{tabular}

Table.1 Sharpness, Image quality and Gap

Table.1 summarizes the outputs of the image dataset fed as input to the conventional and the proposed algorithm that is based on the Retinex and the Ostu's, the results shows that the sharpens of the images set 1 applying the conventional is -55 and the proposed method is 50 , the sharpness observed in the image set $2,3,4$ and 5 for the conventional and the proposed are $-43,-23,-49,-45$ and 40, 45,49,55 respectively. So the observed results shows that the proposed method is better compared to the conventional. In the same way the image quality observed on the image sets proves efficacy of the lad out method. The gap across the real and the virtually real images are very much reduced by the application of the proposed method in processing the images thus minimizing the difficulties in the user immersion of the virtual reality environment. In future the paper it to proceed with the examination on the recognition capability of the humans developing the procedure of the content creation by utilizing the algorithm based on the retinex and the Ostu Method. 
Journal of Innovative Image Processing (JIIP) (2020)

Vol.02/ No. 02

Pages: $77-84$

https://www.irojournals.com/iroiip/

DOI:_https://doi.org/10.36548/jiip.2020.2.002

\section{Conclusion}

The virtual reality technology is a future generation technology, incorporating multiples of methods and technical advancements. Yet the images transmissions in the virtually real environments still experiences low quality images and lower processing speed resulting in the huge difference across the real and the virtually real images. To overcome this difficulties the proposed method in the paper utilizes the retinex and the Ostu to enhance the image sharpness and improve the computing speed. The results observed shows that the laid out method improves the image quality and the processing speed compared to the traditional methods. The future scope of the paper is to examine the recognition capacity of the user through the process of content creation utilizing the algorithm laid out in the paper.

\section{References}

[1] Robertson, George, Mary Czerwinski, and Maarten Van Dantzich. "Immersion in desktop virtual reality." In Proceedings of the 10th annual ACM symposium on User interface software and technology, pp. 11-19. 1997.

[2] Bowman, Doug A., and Ryan P. McMahan. "Virtual reality: how much immersion is enough?." Computer 40, no. 7 (2007): 36-43.

[3] Weiss, Patrice L., and Adam S. Jessel. "Virtual reality applications to work." Work 11, no. 3 (1998): 277-293.

[4] Carrozzino, Marcello, and Massimo Bergamasco. "Beyond virtual museums: Experiencing immersive virtual reality in real museums." Journal of Cultural Heritage 11, no. 4 (2010): 452458.

[5] Akizuki, Hironori, Atsuhiko Uno, Kouichi Arai, Soukichi Morioka, Seizo Ohyama, Suetaka Nishiike, Koichi Tamura, and Noriaki Takeda. "Effects of immersion in virtual reality on postural control." Neuroscience letters 379, no. 1 (2005): 23-26.

[6] Burdea, Grigore C., and Philippe Coiffet. Virtual reality technology. John Wiley \& Sons, 2003.

[7] Fehn, Christoph. "Depth-image-based rendering (DIBR), compression, and transmission for a new approach on 3D-TV." In Stereoscopic Displays and Virtual Reality Systems XI, vol. 5291, pp. 93-104. International Society for Optics and Photonics, 2004.

[8] Urbancsek, Tamás, and Miklós Vogel. "Sensing and Monitoring Methods in Telerobotical Applications Fusing Real-time Video Transmission and Calibrated Virtual Reality." INES 2002, IEEE proceedings (2002): 107-110.

[9] Bindhu, V. "Biomedical Image Analysis Using Semantic Segmentation." Journal of Innovative Image Processing (JIIP) 1, no. 02 (2019): 91-101.

[10] Lee, Youngwoo, and Jin Heon Kim. "A Computational Improvement of Otsu's Algorithm by Estimating Approximate Threshold." Journal of Korea Multimedia Society 20, no. 2 (2017): 163-169. 
Journal of Innovative Image Processing (JIIP) (2020)

Vol.02/ No. 02

Pages: $77-84$

https://www.irojournals.com/iroiip/

DOI:_https://doi.org/10.36548/jiip.2020.2.002

[11] Song, Seung-Keun. "Regulatory reform solution of VR contents industry based on simulator." Journal of the Korea Institute of Information and Communication Engineering 21, no. 11 (2017): 2083-2088.

[12] Ali, Ahmed Hussain, Ali Nihad Abbas, Loay Edwar George, and Mohd Rosmadi Mokhtar. "Image and audio fractal compression: comprehensive review, enhancements and research directions." Indonesian Journal of Electrical Engineering and Computer Science 15, no. 3 (2019): 1564-1570.

[13] Shakya, Subarna. "Virtual Restoration Of Damaged Archeological Artifacts Obtained From Expeditions Using 3d Visualization." Journal of Innovative Image Processing (JIIP) 1, no. 02 (2019): 102-110.

[14] Wen, Shuhuan, Xueheng Hu, Jinrong Ma, Fuchun Sun, and Bin Fang. "Autonomous robot navigation using Retinex algorithm for multiscale image adaptability in low-light environment." Intelligent Service Robotics 12, no. 4 (2019): 359-369.

[15] Zhang, Shu, Ting Wang, Junyu Dong, and Hui Yu. "Underwater image enhancement via extended multi-scale Retinex." Neurocomputing 245 (2017): 1-9.

[16] Manoharan, S. (2019). A Smart Image Processing Algorithm for Text Recognition, Information Extraction and Vocalization for the Visually Challenged. Journal of Innovative Image Processing (JIIP), 1(01), 31-38.

\section{Authors Biography}

Dr. R. Dhaya is currently in the Professor, in Department of Computer science and Engineering at King Khalid University, in Kingdom of Saudi Arabia. His major area of research includes Image and Video Processing Algorithms, Computer Vision, Motion Analysis, Stereo Vision, Object Recognition, computer graphics, photo interpretation, image retrieval, Embedded Image Processing and Real-time image and video processing applications. 